\title{
La fertilización orgánica del melloco (Ullucus Tuberosus L.) y su rentabilidad en la comunidad de Tuncarta
}

\section{The organic fertilization of melloco (Ullucus Tuberosus L.) and its profitability in the community of Tuncarta}

Pablo Antonio Abrigo Córdova

Universidad Internacional del Ecuador, Ecuador

Autor para correspondencia: egtmaloja@gmail.com

Fecha de recepción: 12 de Septiembre de 2016 - Fecha de aceptación: 01 de Noviembre de 2016

Resumen: Se realizó diagnósticos participativos para conocer el estado actual del cultivo de melloco (Ullucus tuberosus L.) en la comunidad indígena de Tuncarta, Ecuador, siendo necesario aplicar grupos focales, entrevistas y encuestas, determinando la producción muy limitada del cultivo. Una vez conocida la realidad se planteó la investigación que tuvo como propósito conocer el comportamiento agronómico del cultivo aplicando dos clases de abono orgánico (humus de lombriz y estiércol de cabra) y para saber la eficacia se empleó un diseño estadístico experimental en bloques al azar, con nueve tratamientos y cuatro repeticiones totalizando 36 unidades experimentales. Las variables evaluadas en el área útil de las parcelas fueron: porcentaje de brotación, días a la emergencia, altura de la planta, diámetro del tallo porcentaje de incidencia de plagas y enfermedades, número de tubérculos por planta, tamaño de los tubérculos y rendimiento por planta, entre otras. Los resultados obtenidos fueron de alta significancia estadística en las siguientes variables: tamaño y número de hojas, número de ramificaciones, número de flores, rendimiento por planta. También se evaluaron variables como el rendimiento por hectárea, en donde el ecotipo rojo con la aplicación de humus de lombriz dio el mejor peso con el tratamiento A1B1 cuyo valor fue de $15949,80 \mathrm{~kg} / \mathrm{ha}$ y el valor más bajo registró ecotipo verde sin fertilización con $4802,70 \mathrm{~kg} / \mathrm{ha}$. También se realizaron los costos de producción para cada tratamiento aplicados al cultivo, donde se obtuvo la mejor producción en el tratamiento 1 arrojando valores en la relación beneficio - costo $(\mathrm{B} / \mathrm{C})$ de 2,19.

Palabras claves: melloco; descriptores agronómicos; producción orgánica; costos de producción; diseño estadístico

Abstract: Paragraph participatory assessment was conducted to know the current state of culture melloco (ullucus L.) in the indigenous community of Tuncarta, Ecuador, still a need for focus groups, interviews and surveys, determining the very limited production product of the crop. Once we know the reality the research was aimed to know the agronomic crop behavior using two kinds of organic fertilizer (humus and goat manure) and para saber effectiveness is used a statistical experimental design in randomized blocks was raised, with nine treatments and four replications totalizing 36 experimental units. The variables evaluated in the useful area of the were plots: Percentage of sprouting, days to emergence, plant height, stem diameter percent incidence of pests and diseases, number of tubers per plant, tuber size and yield per plant, among others. The results were high statistical significance on the following variables: size and number of leaves, number of 
branches, number of flowers, plant performance. Variables such as yield per hectare where the red ecotype with the application of vermicompost had the best weight with A1B1 treatment were also evaluated whose value was $15949.80 \mathrm{~kg}$ / ha and the lowest value ecotype sin Register 4 green with fertilization $802.70 \mathrm{~kg} / \mathrm{ha}$. cost (B / C) 2.19 - production costs para each treatment applied to the crop, where the best production in Treatment 1 was obtained yielding securities in the benefit.

Key words: melloco; agronomic descriptors; organic production; production costs; statistical design

\section{Introducción}

Uno de los terceros objetivos del Plan Nacional del Buen vivir (sumak kawsay) que plantea nuestro país el Ecuador es "Mejorar la calidad de vida de la población" (SENPLADES, 2013). La calidad de vida empieza por el derecho al Buen Vivir: agua, alimentación, salud, educación y vivienda, como requisito para lograr el fortalecimiento de capacidades y potencialidades individuales y sociales.

La Constitución, en el artículo 66, establece "el derecho a una vida digna, que asegure la salud, alimentación y nutrición, agua potable, vivienda, saneamiento ambiental, educación, trabajo, empleo, descanso y ocio, cultura física, vestido, seguridad social y otros servicios sociales necesarios". Por ello, mejorar la calidad de vida de la población es un proceso multidimensional y complejo (SENPLADES, 2013). Entre los derechos para mejorar la calidad de vida se incluyen el acceso al agua y a la alimentación (art. 12).

De alguna manera la utilización de abonos orgánicos en la producción de cultivos representa una mejora en la calidad de vida de los habitantes de nuestro país, específicamente en la producción de melloco, ya que, en el Ecuador es considerado como el segundo tubérculo en importancia luego de la papa. Es parte de la alimentación de la población ecuatoriana de todos los estratos sociales y constituye un componente de los sistemas agrícolas de los pequeños agricultores de la sierra. Es un producto de muy buenas cualidades nutritivas, cuyo cultivo puede adaptarse muy fácilmente a condiciones bioclimáticas diversas y a suelos diferentes. Al igual que la quinua, maíz, oca y muchos otros cultivos autóctonos forman históricamente uno de los principales alimentos del hombre andino (Abrigo, 2007).

Desde el punto de vista socioeconómico es importante, porque el melloco es cultivado con un mínimo uso de insumos químicos, su costo de producción es bajo y accesible para agricultores de escasos recursos económicos. Este cultivo presenta algunas bondades agronómicas que le confieren ciertas ventajas sobre otras que se cultivan en difíciles condiciones andinas, se produce en suelos marginales de baja fertilidad.

Actualmente existe poca producción de este cultivo, por falta de conocimiento de las personas, cambio de hábitos alimenticios producidos en las comunidades debido principalmente a la aculturación, las técnicas deficientes de manejo y consecuentemente el abandono de las tierras para migrar a la ciudad (Espinoza, 1997). De acuerdo a la problemática planteada resultó de gran interés introducir tres ecotipos de melloco y estudiar su comportamiento agronómico, 
conocer su manejo y rentabilidad, proponiendo nuestra práctica y ofrecer una interesante alternativa para nuestros agricultores para producir estos ecotipos.

Para el desarrollo del presente trabajo investigativo se planteó los siguientes objetivos:

\section{Objetivo Primario:}

Evaluar el efecto de la fertilización orgánica en la producción y rentabilidad de tres ecotipos de melloco.

\section{Objetivos secundarios:}

Valorar el comportamiento agronómico de tres ecotipos de melloco, frente a dos clases de abono orgánico en Tuncarta - Saraguro.

Determinar los costos de producción y rentabilidad de tres ecotipos de melloco por cada tratamiento en estudio.

Difundir a los beneficiarios directos e indirectos, las técnicas de producción.

\section{Hipótesis:}

La producción de melloco (Ullucus tuberosus $L$ ) ha disminuido principalmente, por diferentes factores económicos, sociales y culturales en el sector de Tuncarta, lo cual puede ser mejorado si se utilizan abonos orgánicos y se aplican en tres ecotipos de melloco.

Los objetivos e hipótesis de la presente investigación están relacionados directamente, ya que se se utilizarán dos clases de abonos orgánicos (humus y estiércol de animales) y un testigo en la en los terrenos pertenecientes al colegio Agropecuario, cuyos resultados al concluir el ensayo influenciarán directamente en el incremento de la producción al agregarle dos clases de abono orgánico, siendo muy beneficioso para la comunidad de Tuncarta, ya que permitirá recuperar el tubérculo.

\section{Explore la Importancia del problema:}

El problema radica porque existe baja producción de melloco, debido a la aculturación y a la producción limitada, además no aplican técnicas apropiadas de cultivo lo que ocasiona finalmente la pérdida del cultivo. De ahí el interés por saber cuál es el comportamiento agronómico de tres ecotipos de mellocos, frente a dos clases de abono orgánico, conocer su manejo y rentabilidad, proponiendo nuestra práctica y ofrecer una interesante alternativa para nuestros agricultores en este cultivo.

\section{Métodos}

La investigación se efectuó en los terrenos pertenecientes al Colegio Agropecuario "Nuckanchik Kawsay" de la comunidad de "Tuncarta" perteneciente a la parroquia Saraguro del 
cantón Saraguro y provincia de Loja - Ecuador. La localidad posee una altitud $2850 \mathrm{msnm}$; con una temperatura media anual de $8^{\circ} \mathrm{C}$; precipitación anual media de $1100 \mathrm{~mm}$ y con una zona de vida bosque seco montano bajo (bs - MB), con vientos predominantes de este a oeste, con una humedad relativa del $65 \%$.

En éste trabajo investigativo se utilizaron los siguientes materiales: Material Vegetal: Semillas de tubérculos de mellocos: ecotipos, rojo, blanco y verde. Materiales de Campo:

Tractor agrícola, yunta, piola, ceniza, abono orgánico, estacas de madera, herramientas agrícolas.

El lugar donde se desarrolló la investigación está ubicado al norte de la provincia de Loja aproximadamente a $73 \mathrm{~km}$ de la cabecera provincial y posee las siguientes características:

UTM: $698342,7133 \quad 9599302,4374$

Área de intervención: 376581,132614 metros cuadrados

Perímetro: 2462,510922 metros

\section{Coordenadas del área de influencia:}

$\begin{array}{ll}\text { P1: } 698.258,445 & 9.599 .063,677 \\ \text { P2: } 698.254,249 & 9.599 .633,413 \\ \text { P3: } 698.918,305 & 9.599 .633,413 \\ \text { P4: } 698.918,979 & 9.599 .067,981\end{array}$

Rango altitudinal del área de influencia de sur a norte: 2850 a los 2.280 m.s.n.m.

Clima: Templado lluvioso

En la figura 1. Se observa las principales características limítrofes. 


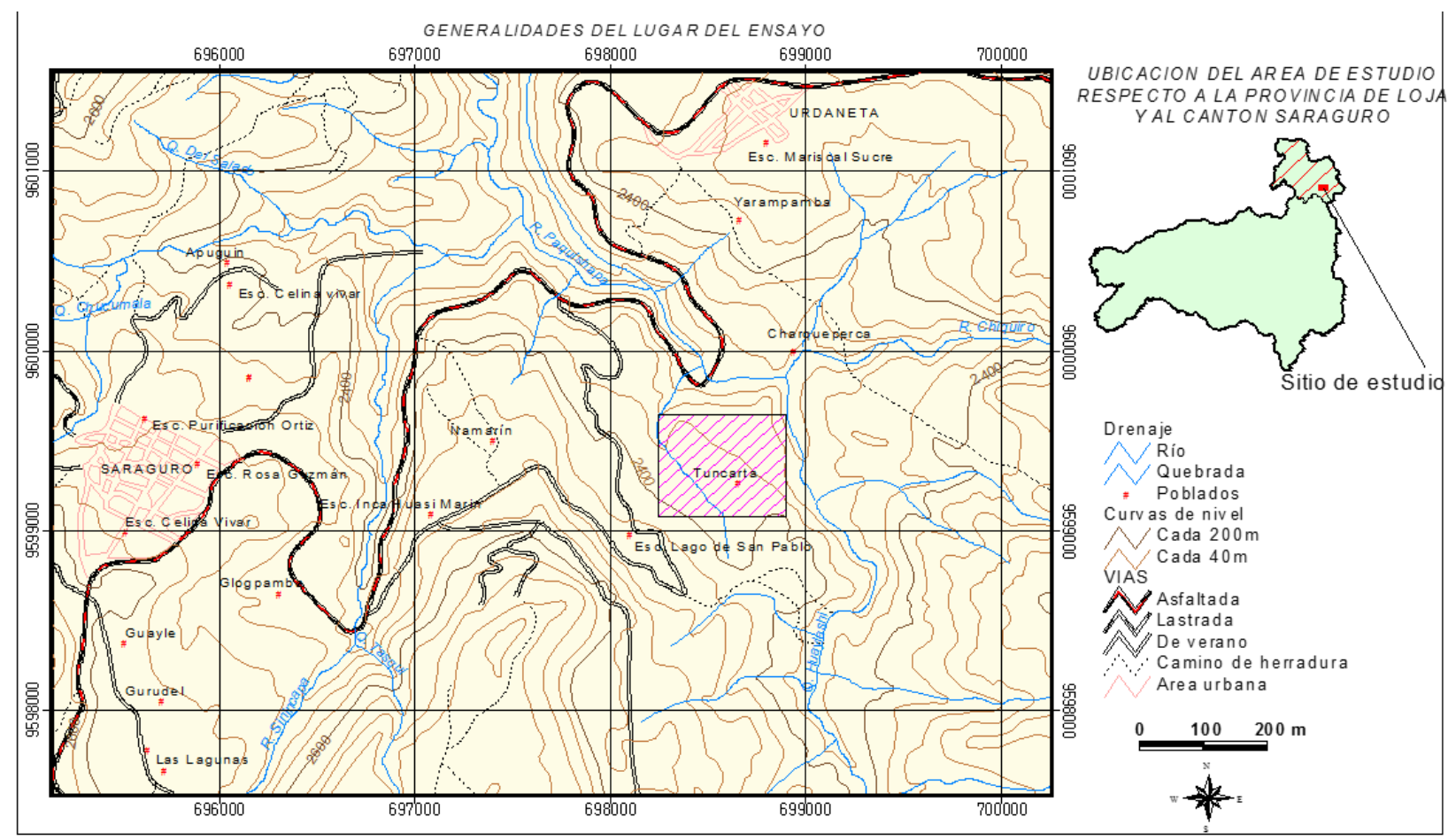

Figura 1. Sectorización del lugar donde se desarrolló la investigación.

Fuente: El autor

Al norte limita con el barrio Oñakapac.

Al sur con el cantón Saraguro.

Al este con la parroquia Urdaneta.

Al oeste con el barrio Namarin.

La metodología utilizada se refirió al comportamiento agronómico de tres ecotipos de melloco (variedades roja, verde y blanco; semilla introducida proveniente del cantón Salcedo) mediante la aplicación de dos abonos orgánicos (humus de lombriz y estiércol de cabra) frente al testigo ( $\sin$ abono); previo a ello se hizo un sondeo a los agricultores de la comunidad para conocer el estado inicial de la producción del cultivo; proporcionando como resultado, que los moradores del sector cultivan solamente para el autoconsumo sin ningún tipo de tecnología para el manejo del cultivo.

Las variables a estudiar fueron las siguientes:

\section{Análisis estadístico}

El diseño estadístico fue en Bloques al Azar, con nueve tratamientos y cuatro repeticiones totalizando 36 unidades experimentales. Las variables evaluadas en el área útil de las parcelas fueron: porcentaje de brotación, días a la emergencia, altura de la planta, diámetro del tallo principal, altura de la primera ramificación, número de ramificaciones, forma y tamaño de las hojas, número de hojas por planta, color y forma de la flor, pétalos y sépalos, clase de inflorescencia, días a la floración fisiológica, número de flores por planta, porcentaje de incidencia de plagas y enfermedades, número de tubérculos por planta, tamaño de los tubérculos 
(largo y ancho) y rendimiento por planta. Los resultados obtenidos fueron de alta significancia estadística en las siguientes variables: tamaño y número de hojas, número de ramificaciones, número de flores, rendimiento por planta.

Para la implantación del cultivo primeramente se reconoció el terreno, luego se lo preparó, se construyeron y trazaron las parcelas y se las codificó (figura 1), se abonó antes y después de la siembra, se sembró y se cosechó.
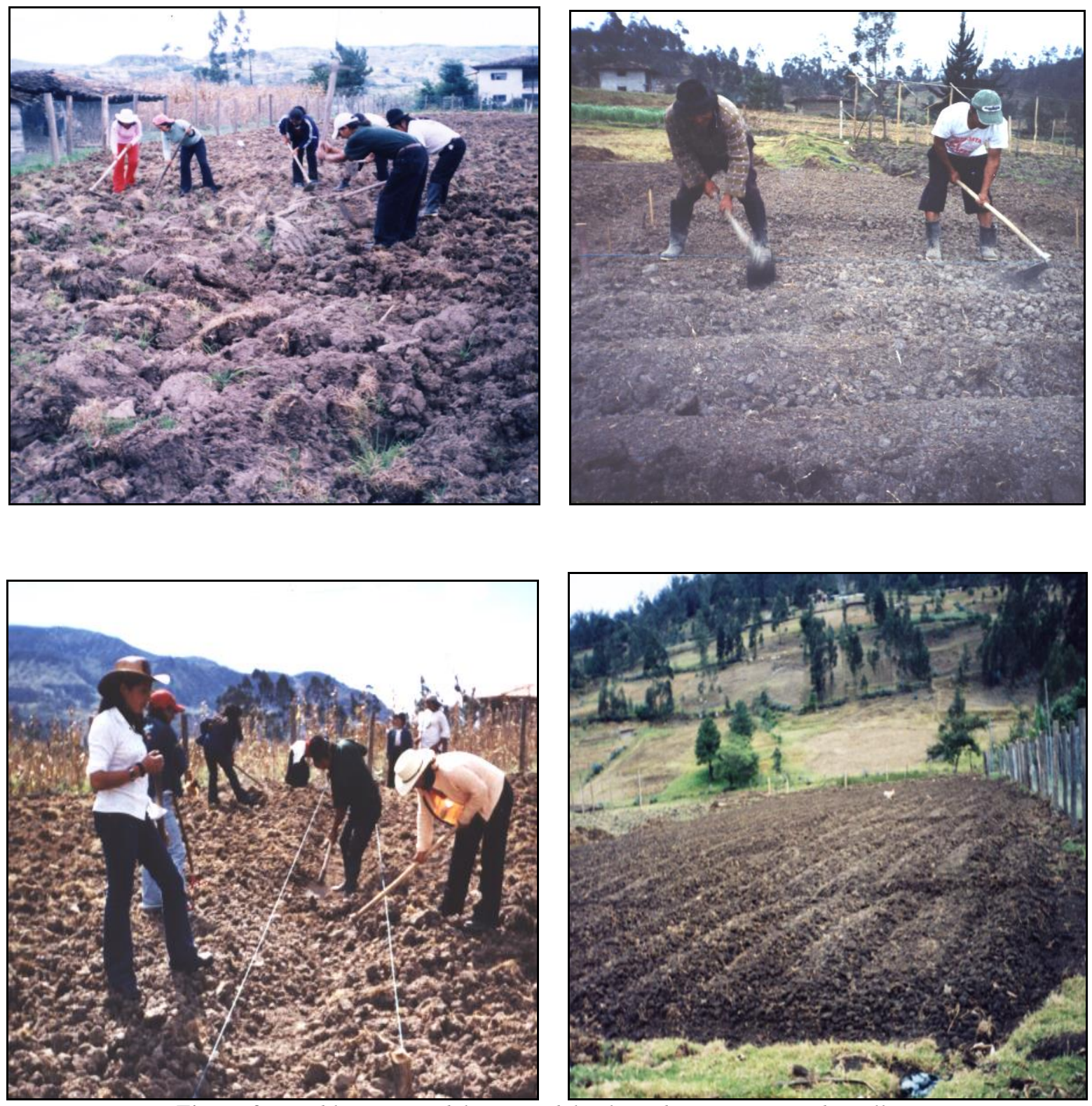

Figura 2. Establecimiento del ensayo del cultivo de tres ecotipos de melloco

Fuente: El autor 


\section{Diseño de la Investigación}

Se utilizó el diseño experimental bifactorial, conducido con bloques al azar con nueve tratamientos y cuatro repeticiones (tabla 1.), y para establecer el o los mejores tratamientos se recurrió a la prueba de rangos múltiples de Duncan, con un nivel de significancia del 0,05. El modelo estadístico fue el siguiente:

$$
Y_{i j k}=\mu+\alpha_{i}+\beta_{j}+(\alpha \beta)_{I J}+Y_{K}+\varepsilon_{i j K}
$$

De donde:

Yijk = Eficiencia de los tratamientos

$\mu=$ Gran media del experimento

cijK, = Efecto del error experimental

$\alpha i=$ Efecto de las diferentes dosis de abono orgánico

$\beta \mathrm{j}=$ Efecto de los ecotipos

$\mathbf{Y K}=$ Efecto de las repeticiones

i = 1 y 2 abonos orgánicos; $\mathrm{j}=1,2,3$ ecotipos y $\mathrm{k}=1,2,3$ y 4 repeticiones

\section{El análisis de varianza}

Tabla 1. Análisis de varianza del ensayo

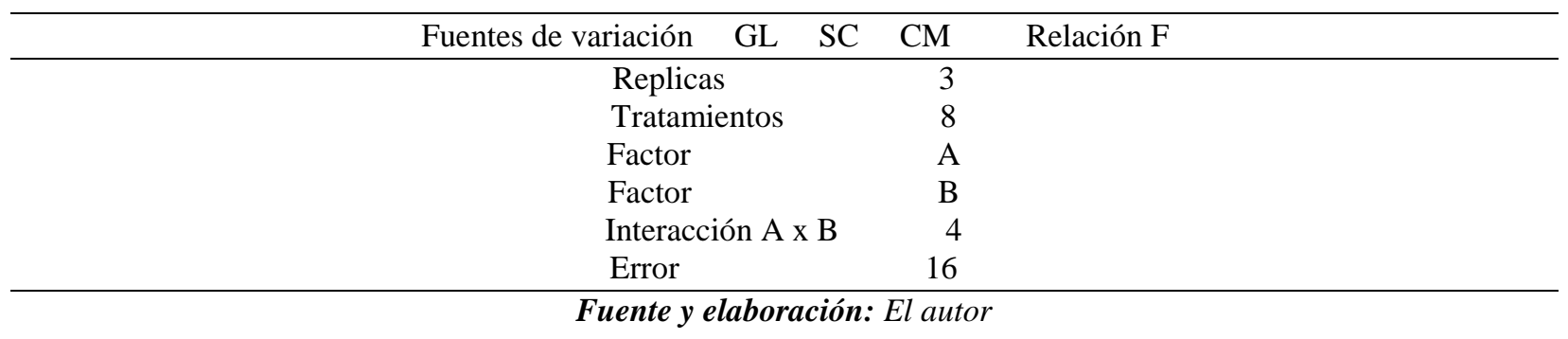

Tabla 2. Tratamientos utilizados para evaluar el rendimiento por planta.

\begin{tabular}{llll}
\hline \multicolumn{2}{l}{ Tratamientos } & Ecotipo más abono & Código \\
\hline 1 & Melloco Rojo + Humus & A1B1 \\
2 & Melloco Rojo + Abono de cabra & A1B2 \\
3 & Melloco Blanco + Humus & A2B1 \\
4 & Melloco Blanco + Abono de cabra & A2B2 \\
5 & Melloco Verde + Humus & A3B1 \\
6 & Melloco Verde + Abono de cabra & A3B2 \\
7 & Melloco Rojo + Testigo & A1T0
\end{tabular}




\begin{tabular}{ccc}
8 & Melloco Blanco + Testigo & A2T0 \\
9 & Melloco Verde + Testigo & A3T0 \\
\hline
\end{tabular}

Para evaluar las variables, es necesario señalar que se tomaron datos de 12 plantas de los surcos centrales por cada parcela y se obtuvo un promedio. Se evaluaron las variables dependientes (tratamientos) e independientes (Producción) (figura 2).

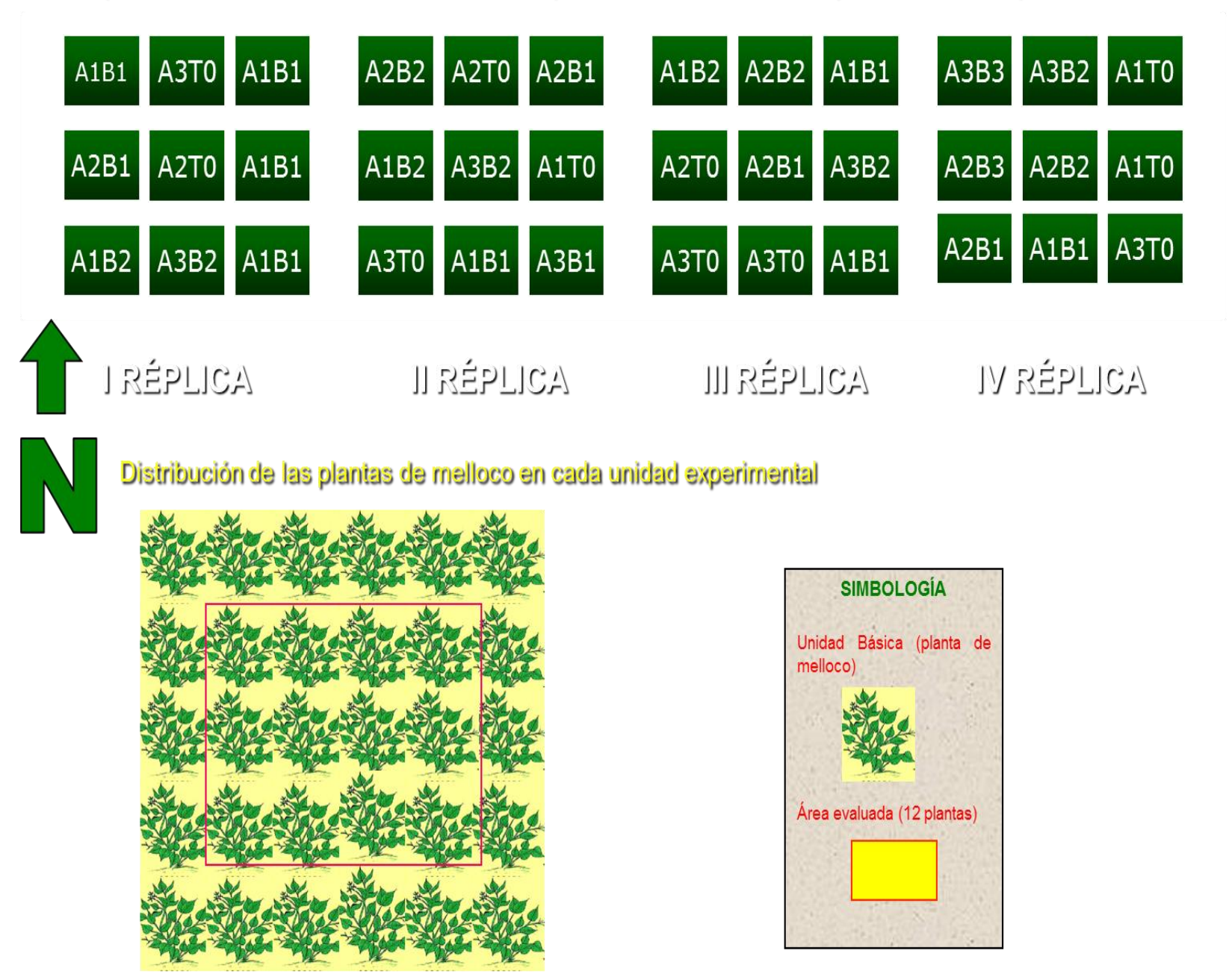

Figura 3. Croquis de distribución de las unidades experimentales

Fuente y elaboración: El autor

El rendimiento por planta se lo hizo de acuerdo al número total de plantas de los tres ecotipos por separado. Con los datos obtenidos del rendimiento por planta de los tres ecotipos en estudio se realizó una proyección para una hectárea de acuerdo al número de plantas, lo que permitió establecer el rendimiento promedio por hectárea.

La rentabilidad de los tres ecotipos de melloco se determinó de acuerdo a los rendimientos por planta y por hectárea, estableciendo los costos de producción de cada tratamiento del cultivo y el tratamiento más rentable por cada ecotipo, además se realizó el análisis financiero del cultivo con una proyección hasta el quinto año. 


\section{Características de los participantes:}

La participación de los moradores de la comunidad de Tuncarta y estudiantes del colegio fue fundamental para la realización de la investigación; ya que, participaron activamente en las labores culturales y en la socialización de los resultados.

\section{Resultados y Discusión}

\section{Primer objetivo}

\section{a) Porcentaje de brotación}

El ecotipo verde presentó el mayor porcentaje de brotación en un $87 \%$, luego en orden descendente aparecen los ecotipos rojo con $60 \%$ y el con $59 \%$; los días a la emergencia se dieron con mayor rapidez en el ecotipo verde con 19 días (figura 4); en cambio los que tardaron más este proceso fueron los ecotipos rojo y blanco con 28 y 24 días respectivamente. Esto se debió a que en la zona se presentó un periodo seco, para ello fue necesario realizar riegos localizados en una cantidad de $1000 \mathrm{ml}$ por planta cada dos días y así se compenso éste déficit.

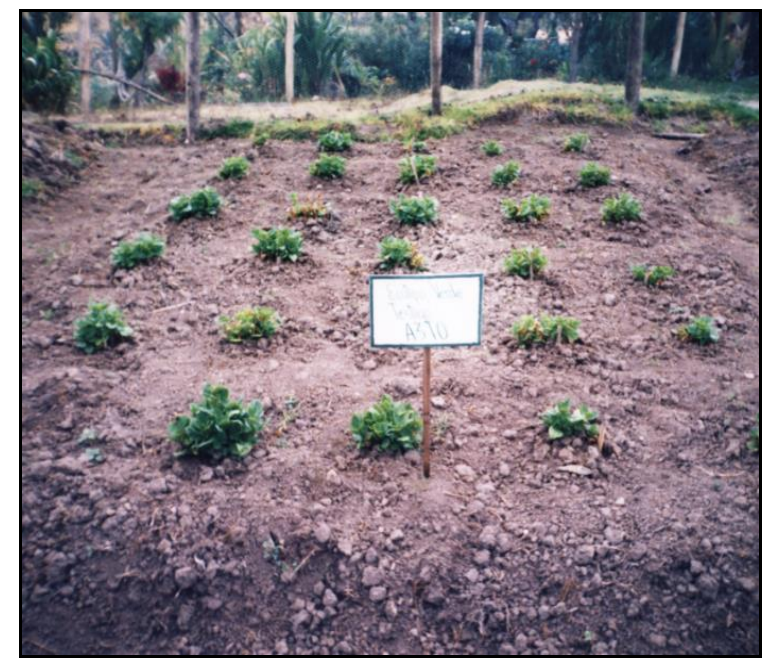

a) Ecotipo verde 


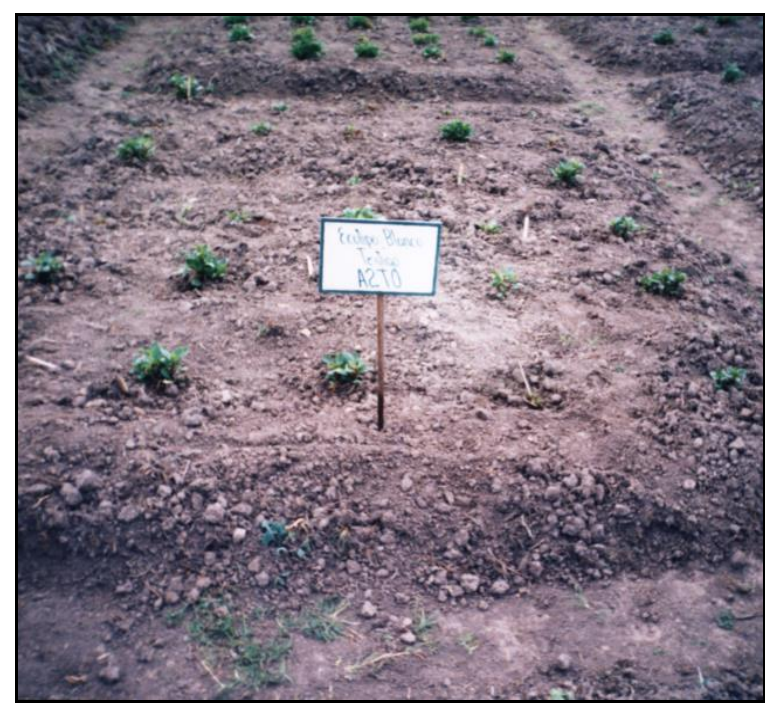

b) Ecotipo rojo

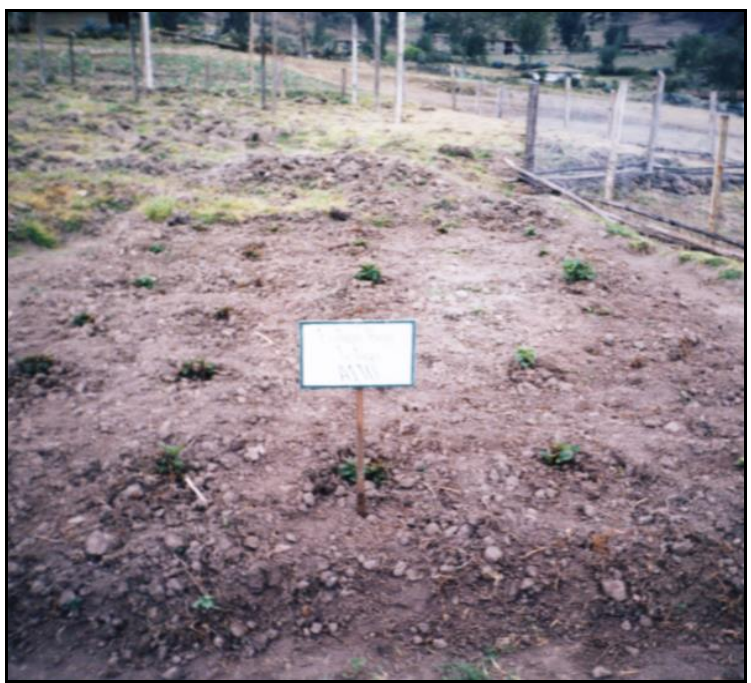

c) Ecotipo blanco

Figura 4. Emergencia de los ecotipos de melloco.

Fuente: El autor

\section{c) Días a la floración}

Los días a la floración se dio a los 98 días en el ecotipo blanco, a los 110 días en el ecotipo rojo y 130 días en el ecotipo verde; evidenciándose que el ecotipo verde es el más temprano. Los días a la tuberización (formación de los tubérculos) se dieron a los 122, 115 y 143 días para los ecotipos rojo, blanco y verde respectivamente, demostrándose que el ecotipo blanco fue el más metabólicamente activo (figura 5). 

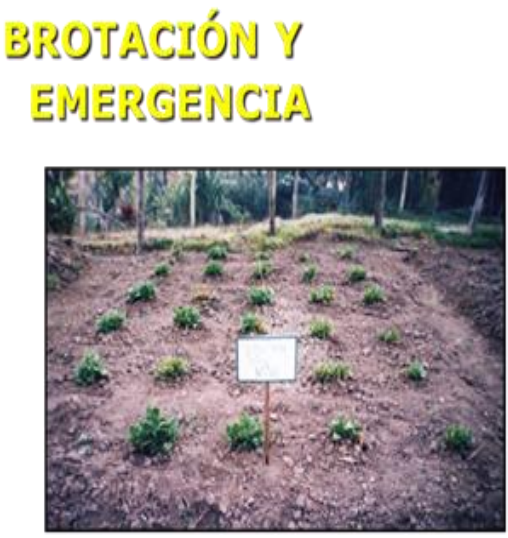

Ecot̉jpo vercte $(37-19)$

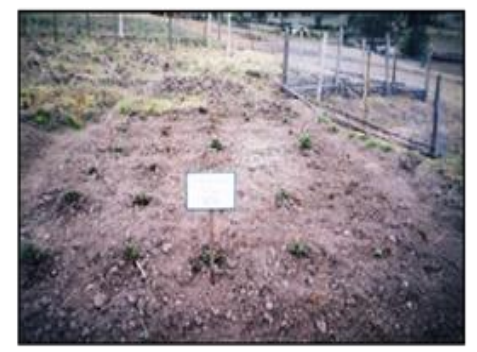

Ecotjpo rojo (50 y 23 )

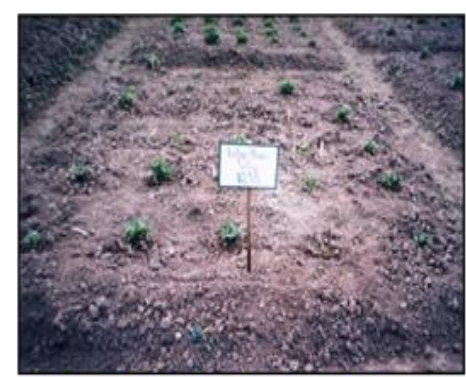

Ecobjpo blassco $(59$ y 2 3

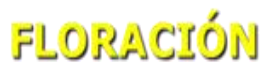

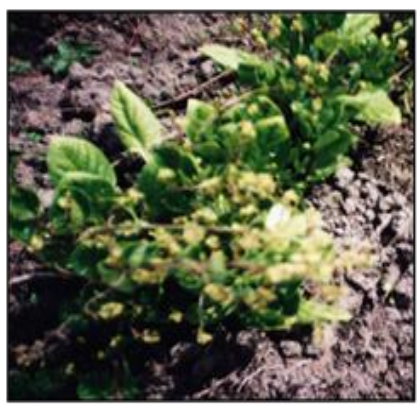

Ecobjpo yercte (130)

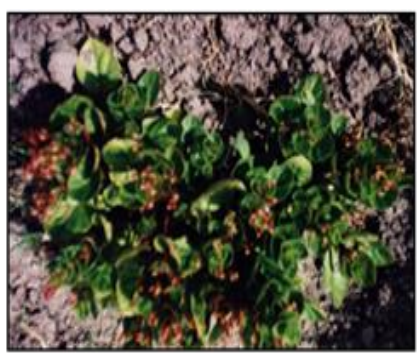

Ecotjipo rojo (110)

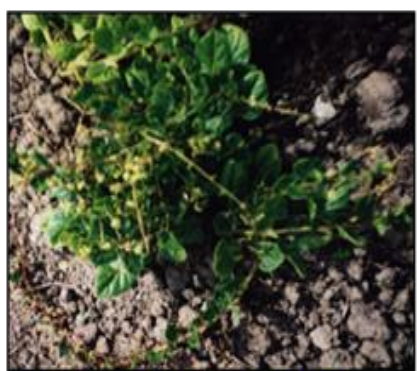

Ecotjpo blanso (93)
COSECSTA

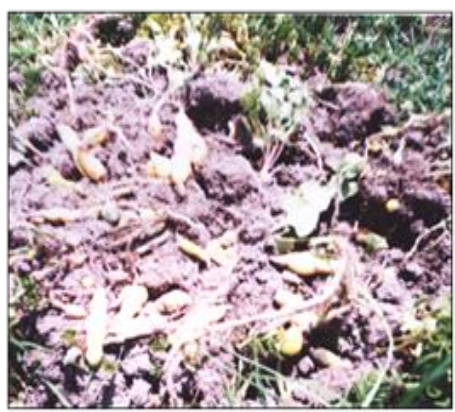

Ecotjpo yercte (225)

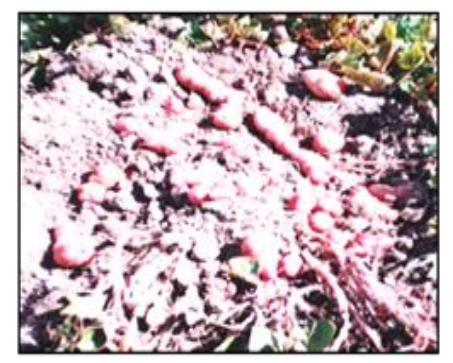

Ecotjpo rojo (210)

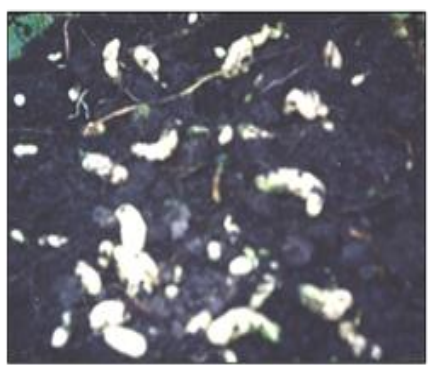

Ecotjpo blanso (130)

Figura 5. Días a la brotación, emergencia y cosecha del cultivo de melloco.

Fuente: El autor

\section{d) Fertilización orgánica}

Se utilizó como abonos orgánicos el excremento de cabra, procedente del cantón Zapotillo y el humus de lombriz proveniente del Municipio de Loja. Una vez conocidos los macronutrientes en el suelo del sector, se empleó 500 gramos por planta de abono de cabra y 550 gramos por planta de humus de lombriz. Estas dosis se colocaron fraccionadas, un $50 \%$ antes de la siembra con 15 días de anticipación a una profundidad de $15 \mathrm{~cm}$. Y el otro $50 \%$ se aplicó a los tres meses de haber sido implantado el cultivo (figura 6). 

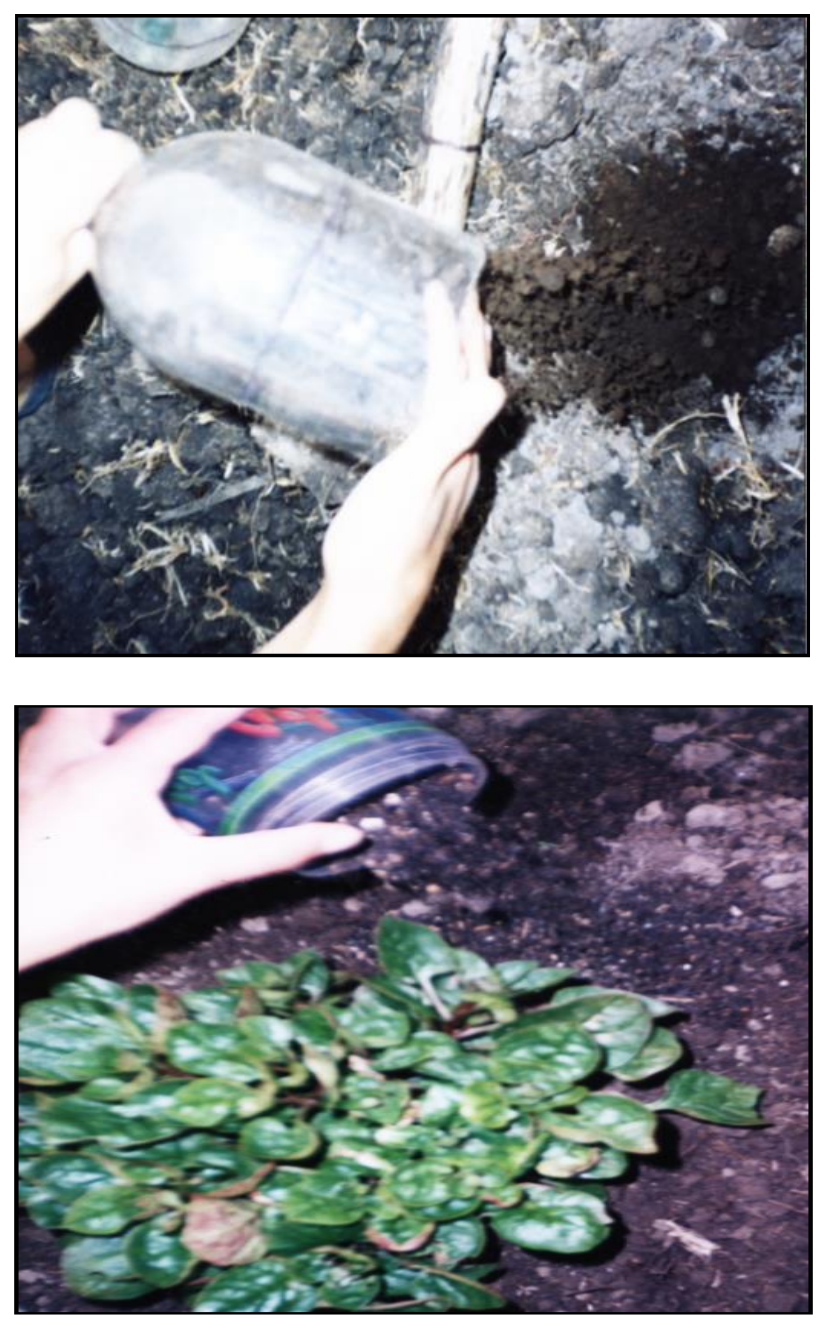

Figura 6. Colocación de abono orgánico antes y después de la siembra

Fuente: El autor

\section{e) Días a la cosecha}

Los días a la cosecha, el ecotipo blanco presentó 180 días, seguido de los ecotipos rojo y verde con 210 y 225 días respectivamente (figura 7).

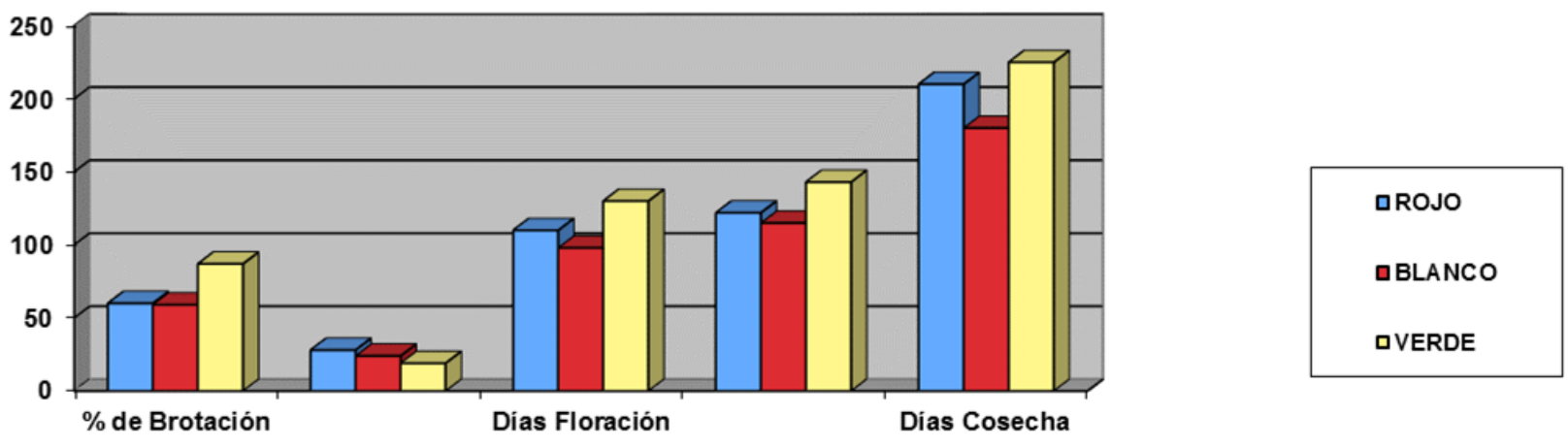

Figura 7. Porcentaje de brotación, días a la emergencia, floración, tuberización y cosecha del cultivo de melloco. Fuente y elaboración: El autor 


\section{f)Características Morfológicas}

Las hojas, el ecotipo blanco y rojo fueron de color verde, con formas carnosas alternas cordana; en cambio el ecotipo verde fue de color verde púrpura, con forma alterna cordada.

Por otra parte el tallo, para los ecotipos blanco y verde, fue de color verde y la forma triangular carnosa y cilíndrica respectivamente; el ecotipo rojo color verde púrpura y la forma triangular carnosa.

El color del fruto de los ecotipos blanco, rojo y verde fueron, blanco, rojo y verde en su orden y su forma varía entre falcado a ovalado falcado y falcado respectivamente.

Los sépalos presentaron la forma alargada en todos los ecotipos; y su color varía de amarillo con tintes púrpura en el ecotipo blanco a púrpura en el rojo y amarillo en el verde. Los pétalos son opuestos a cada estambre, existiendo además cinco estambres pequeños, presentando formas que varían de alargado retorcido en el ecotipo blanco a alargado en los ecotipos rojo y verde.

Las flores se componen de un perigonio de cinco tépalos, al centro de la flor sobresale un ovario súpero ovoide y globoso que termina en un estigma redondeado. El color de la flor del ecotipo blanco y verde fue amarillo, el ecotipo rojo amarillo con franja púrpura y sus formas fueron de estrella en todos los casos. En lo que tiene que ver con la inflorescencia, se observó que su color es amarillo y su forma es de racimo axilar para todos los ecotipos.

\section{g) Peso de los tubérculos}

Comparados los promedios a través de la prueba de rangos múltiples de Duncan, existe diferencia altamente significativa entre tratamientos, réplicas; así como los correspondientes a los factores, ecotipos y abonos. Esta diferencia está representada especialmente en el peso de obtenido en el tratamiento 1 con 930,42 gramos por planta, obteniéndose el valor más alto, seguido por el tratamiento 9 que es el más bajo con 280 g. con 855,70 g. (figura 8 ). El abono orgánico que dio mejores resultados al aplicarlo al cultivo, fue el humus de lombriz, contiene ácidos húmicos, fúlvicos de cadena larga con enlaces orgánicos unidos a los macronutrientes. Las posibles diferencias existentes en la elaboración de éste abono en comparación con el abono de cabra, es que se emplean una diversidad de materiales orgánicos en la aplicación en el campo. 


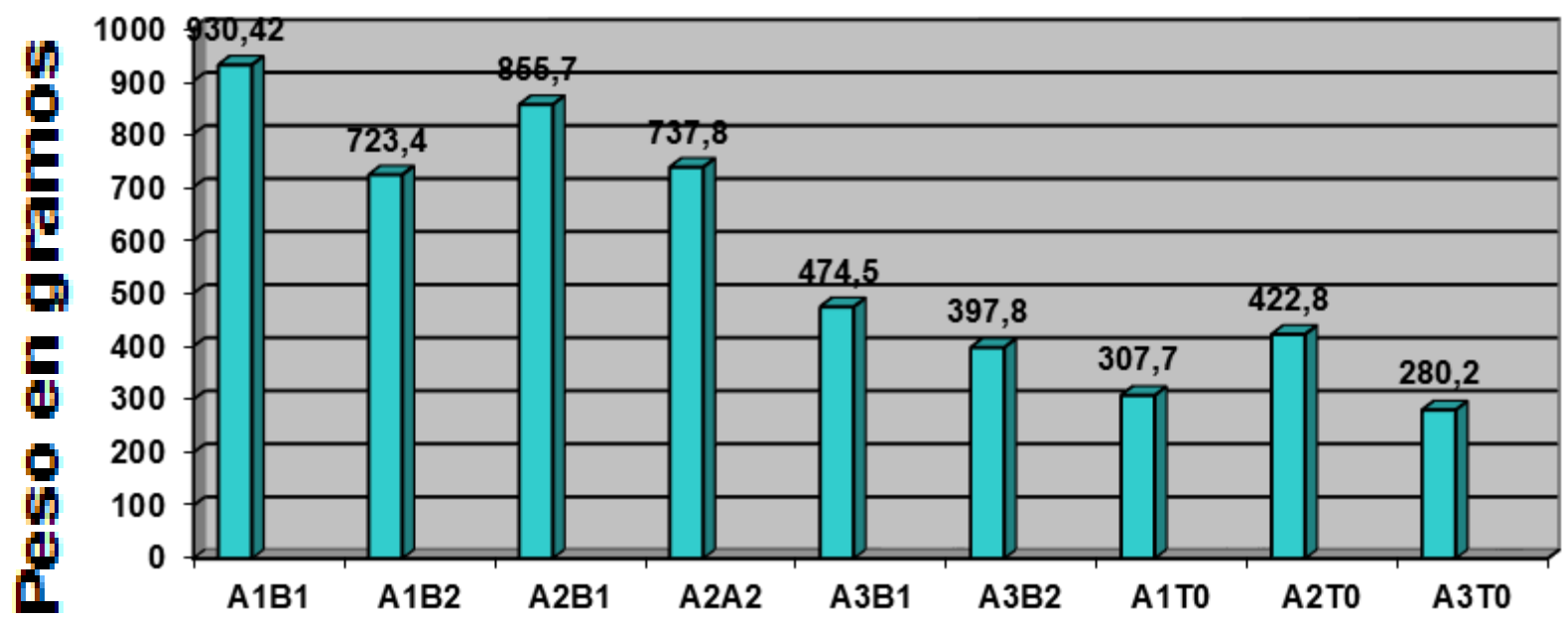

Figura 8. Peso de los tubérculos en los nueve tratamientos aplicados al cultivo de tres ecotipos de melloco Fuente y elaboración: El autor

\section{h) Control fitosanitario}

El seguimiento, evaluación y control de las principales plagas se las verificó en el transcurso del cultivo. Encontrándose en estado adulto pulguillas (Epitrex cucumeris) y diabroticas (Diabrotica longicornis), presentando una incidencia del 60,6\% y 40,5\% respectivamente. La incidencia se determinó tomando en cuenta el número de plantas sanas e infectadas por tratamiento, siendo para ello aplicar productos ecológicos como el MATCH en dosis de 1 cc por litro de agua. También se presentó el cutzo (Barotheus sp.) en estadio II, aplicándose también productos ecológicos como el BAUVERIL WP en una dosis de 2 cc por litro de agua. La aparición de estos lepidópteros fue en la fase de inicio, intermedia e inicio a la tuberización, efectuándose dos aplicaciones en todo el cultivo. No hubo presencia de enfermedades, ya que se presentó un medio ambiente desfavorable y por ser una especie nueva en la zona, es por ello que no existió ningún tipo de hongos fitoparásitos.

\section{Segundo Objetivo}

\section{i) Rendimiento y rentabilidad}

El rendimiento de los ecotipos con la aplicación de humus para el ecotipo rojo y blanco fue de $15949,80 \mathrm{~kg} / \mathrm{ha}$ y $14669,10 \mathrm{~kg} / \mathrm{ha}$ respectivamente; en cambio con la adición de abono de cabra los rendimientos fueron de $12400,17 \mathrm{~kg} / \mathrm{ha}$ y $12647,70 \mathrm{~kg} / \mathrm{ha}$. Los altos valores obtenidos se debieron al suministro de nutrientes existentes en los abonos orgánicos, condiciones físicas y temperatura adecuada así como la buena calidad de la semilla.

Para determinar la rentabilidad del cultivo de melloco se procedió a calcular los costos de producción por cada tratamiento, durante el primer año, relacionando con los rendimientos obtenidos por cada año, en la tabla 3 se presenta se presenta un ejemplo de cálculo para los tratamientos 1 y 9 .

Tabla 3. Costos y beneficios por año de los tratamiento 1 y 9 aplicados al cultivo de tres ecotipos de melloco. 


\begin{tabular}{lcc}
\hline Rubros & \multicolumn{2}{c}{ Tratamientos 1 y 9 (USD) } \\
& A1B1 & A3T0 \\
\hline Costo del Año 1 & 76,88 & 64,31 \\
Costo del Año 2 & 32,08 & 44,76 \\
Beneficios del año 1 y 2 & 94,90 & 26,89 \\
& & \\
\hline
\end{tabular}

\section{Fuente y elaboración: El autor}

Los análisis financieros del establecimiento del cultivo de melloco para el tratamiento 1 , la tir indica que el máximo interés que se puede pagar por capital utilizado es de 888,25\% (tabla iii), lo que significa que es un tratamiento altamente rentable diferenciándolo de todos los otros, además la relación b/c de 2,19 confirma la rentabilidad; el punto de equilibrio se alcanza en el primer año; por otra parte el valor obtenido en el tratamiento 9 es el menor de todos y no es rentable, puesto que la relación b/c no supera a la unidad, lo que implica que la tir nos arroje valores negativos (figura 9).

Tabla 4. Análisis financiero de la producción de los tratamientos 1 y 9 aplicado al cultivo de tres ecotipos de melloco.

\begin{tabular}{llc}
\hline Rubros & \multicolumn{2}{c}{ Tratamientos 1 y 9 (USD) } \\
& A1B1 & \\
& \multicolumn{2}{c}{ A3T0 } \\
\hline TIR \% & 888,25 & $-350,28$ \\
VAN 12 \% & 186,45 & $-81,87$ \\
B/C & 2,19 & 0,54 \\
PE AÑO 1: \% & 51,04 & $-25,98$ \\
AÑO 2: \$ & 15,37 & $-70,977$ \\
TRC & $3,8(3$ años, 9meses y 29 días & 0,52 (6 meses y siete días) \\
\hline
\end{tabular}

Fuente y elaboración: El autor

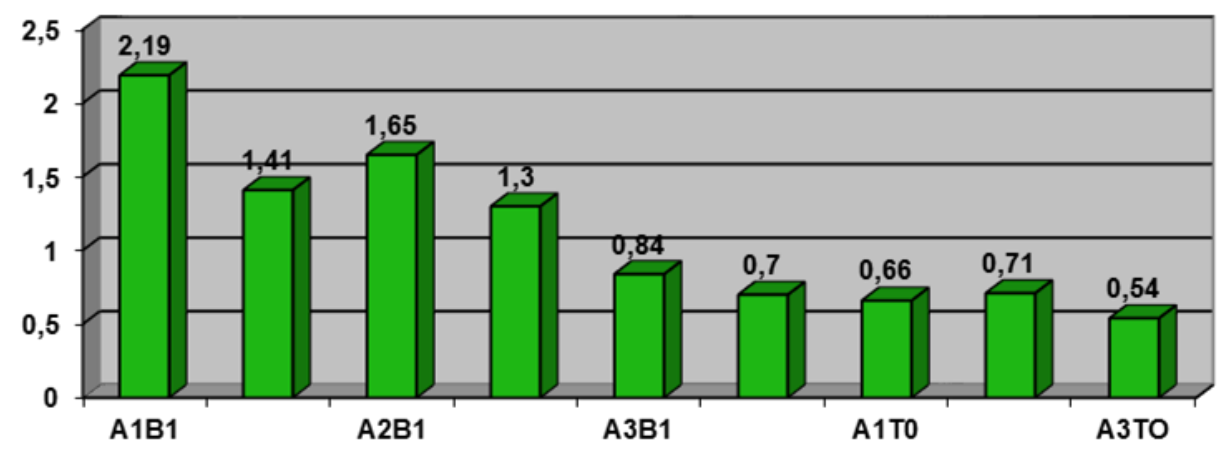

口Relación B/C

Figura 9. Relación $\mathrm{B} / \mathrm{C}$ en los nueve tratamientos aplicados al cultivo de tres ecotipos de melloco, Tuncarta

La socialización de los resultados se efectuó en la comunidad en Tuncarta, existiendo un gran interés tanto de los agricultores como de los consumidores por que se fomente la producción orgánica del cultivo de melloco en la zona.

\section{Tercer Objetivo}


Para la transferencia de tecnología a los beneficiarios directos e indirectos, se efectuó un evento: como el reconocimiento del terreno para la siembra, establecimiento del cultivo, día de campo (figura 10) y la difusión de los resultados en el sector de Tuncarta. Los cuales se desarrollaron a través de la extensión participativa siguiendo una matriz de programación.
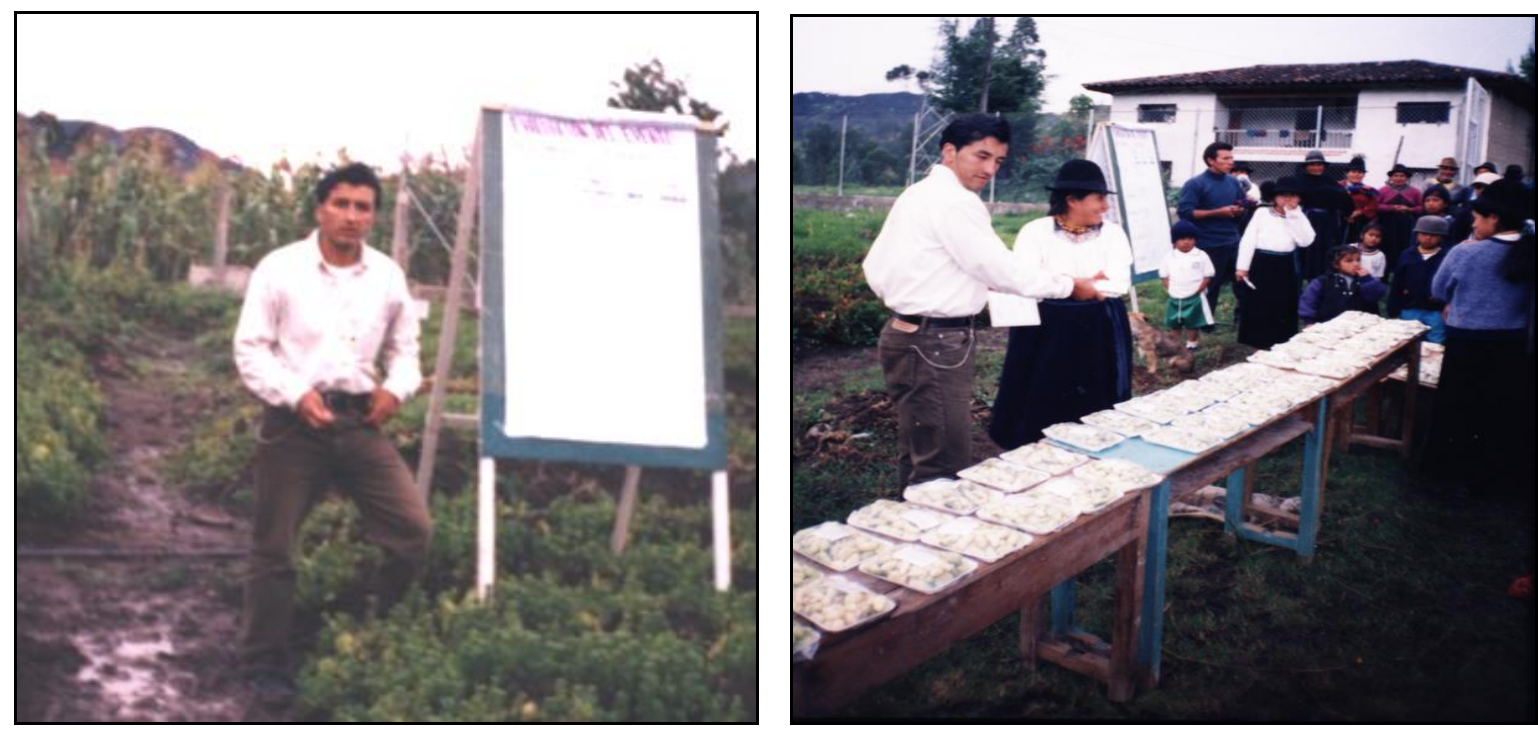

Figura 10. Exposición de los resultados del y producto cosechado en el día de campo. Tuncarta.

\section{Conclusiones}

Los ecotipos rojo y blanco fueron más susceptibles al ataque de plagas (diabrotica y pulguilla) y a la presencia de heladas; en cambio el ecotipo verde fue más resistente.

El ciclo del cultivo (siembra - cosecha) del ecotipo rojo fue de 210 días, el ecotipo blanco 180 días, mientras que el ecotipo verde su ciclo fue de 225 días.

El mejor rendimiento se obtuvo en el tratamiento 1 (A1B1 Ecotipo rojo más humus de lombriz) con 930,42 g/planta y $15949,80 \mathrm{~kg} / \mathrm{ha}$ y el más bajo en el tratamiento 9 (A3T0 Ecotipo verde más testigo) con $280,20 \mathrm{~g} /$ planta y $\quad 4802,70 \mathrm{~kg} / \mathrm{ha}$.

Desde el punto de vista económico, la producción del cultivo de melloco presenta buena rentabilidad si se aplican abonos orgánicos especialmente el humus de lombriz. Sin embargo factores como: bajo precio del producto en el mercado, poca importancia del cultivo en las zonas donde poco o casi nada se cultiva y escasa concientización de los consumidores que no dan importancia a los productos autóctonos, reducen el margen de rentabilidad del cultivar de melloco.

El tratamiento 1 (A1B1 Ecotipo rojo más humus de lombriz) es el más rentable, ya que la relación beneficio - costo $(\mathrm{B} / \mathrm{C})$ supera en 1,19 a la unidad.

Para lograr una mayor producción y rentabilidad del cultivo se sugiere seguir utilizando abonos orgánicos, estableciendo densidades de siembra menores a las expuestas en el presente trabajo investigativo $(0,60 \mathrm{~cm}$ entre planta y $0,80 \mathrm{~cm}$ entre surco). 
También es importante introducir nuevas variedades al sector, siendo motivo de estudio.

En base a los resultados obtenidos en el trabajo investigativo se recomienda utilizar el tratamiento 1 (A1B1 ecotipo rojo con humus de lombriz) ya que registró diferencias significativas en comparación con los demás tratamientos.

\section{Recomendaciones}

Para lograr una mayor producción y rentabilidad del cultivo se sugiere seguir utilizando abonos orgánicos, estableciendo densidades de siembra menores a las expuestas en el presente trabajo investigativo.

También es importante introducir nuevas variedades al sector, siendo motivo de estudio.

Utilizar el tratamiento 1 (A1B1 ecotipo rojo con humus de lombriz) ya que registró diferencias significativas en comparación con los demás tratamientos.

\section{Agradecimiento}

De nosotros, especial testimonio de reconocimiento a la comunidad de Tuncarta, especialmente a los Directivos del Colegio, por la colaboración al brindarnos las facilidades para el desarrollo de la investigación en mención y a todos que de alguna manera impulsaron para que se tenga el éxito deseado.

\section{Bibliografía}

Abrigo, P. (2007). Producción y procesamiento para la comercialización de tres ecotipos de melloco en Saraguro. Tésis Ing. Agr. Loja, Ec., Universidad Nacional de Loja. Área Agropecuaria y de Recursos Naturales Renovables. 233 p.

Abrigo, P., (2012). Los cultivos autóctonos en el sector de tuncarta del cantón saraguro y su perspectiva de rescate como aporte al desarrollo comunitario. Tésis Mgs. Loja, Ec., Universidad Nacional de Loja. Área Jurídica, Social y Administrativa.

Apolo, V. (2000). Guía técnica: Procesamiento y/o transformación de alimentos. Área de Pequeñas Industria. AARNR, UNL. 30 p.

Espinosa, P; C. Crissman. (1997). Raíces y tubérculos andinos: Consumo, Aceptabilidad, Procesamiento. Departamento de Ciencias Sociales (CIP). Editorial Abya-Yala. Quito, Ecuador. 135 p.

Robles, E. (1981). Origen y Evaluación de la oca, ulluco y mashua. Centro de Informática para la Investigación Agrícola. Lima, Perú, Universidad Agraria La Molina, Lima, p. 17. 
Ramon, S. (2012). Influencia lunar en la brotación de tubérculos de melloco (Ullucus tuberosus). Tesis Ing. Agrop. Universidad Técnica Estatal de Quevedo. Facultad de Ingeniería Agropecuaria.

Secretaria Nacional de Planificación y Desarrollo. (2013). Plan nacional del buen vivir. Quito, Ecuador. 600 pp.

Sigcho, M. (2015). Redes de alimentos y producción artesanal en la parroquia Saraguro, cantón Saraguro: Un aporte al análisis de la soberanía alimentaria. 2015, 77 pp.

Vimos, C. (1981). Caracterización y evaluación preliminar agronómico de 90 entradas de melloco, 48 de oca y 36 de mashua del banco de germoplasma del INIAP. Tesis Ing. Ag. Riobamba, Escuela Politécnica del Chimborazo, Facultad de Ingeniería Agronómica. 288 p 